\title{
Trace States and KMS States for Approximately Inner Dynamical One-Parameter Groups of *-Automorphisms
}

\author{
Palle E. T. Jørgensen ${ }^{\star}$ \\ Department of Mathematics, University of Pennsylvania, Philadelphia, PA 19174, USA
}

\begin{abstract}
The usual definition of approximately inner one-parameter groups of *-automorphisms of $C^{*}$-algebras (approximately inner dynamical oneparameter groups) contains a slight asymmetry. When this asymmetry is "corrected", we show that if an approximately inner dynamical one-parameter group has $K M S$ states for one value of inverse temperature $\beta=1 / k T$, then it has $K M S$ states for all values of $\beta$. By the Powers-Sakai Theorem it is enough to show that there is a trace state. We obtain a trace state as a limit of a sequence of "vector states" with respect to a given $K M S$ state and thus solve a problem raised in [6].
\end{abstract}

\section{Introduction}

In the global and axiomatic approach to statistical mechanics [12] the algebra of observables is a $C^{*}$-algebra $\mathfrak{A}$ with unit, and the dynamics $\left\{\alpha^{t}:-\infty<t<\infty\right\}$ is given by a strongly continuous one-parameter group of $*$-automorphisms of $\mathfrak{A}$. The dynamical group $\alpha^{t}$ is said in [9] to be approximately inner if there exists a sequence of hermitian elements $\left(h_{n}\right)$ in $\mathfrak{A}$ such that for all $a \in \mathfrak{U}$ and $t \in \mathbb{R}$ we have

(1) $\left\|e^{i t h_{n}} a e^{-i t h_{n}}-\alpha^{t}(a)\right\| \rightarrow 0$ as $n \rightarrow \infty$.

If $\alpha^{t}$ is assumed strongly continuous, then it follows from the Trotter-Kato Theorem ([3,15] and [17]) that the convergence in (1) is uniform for $t$ in compact subintervals of the real line. If on the other hand the convergence is assumed uniform for $t$ in compact intervals, then $\alpha^{t}$ is automatically strongly continuous, as can be checked by a $2 \varepsilon$ argument.

We suggest adding one minor technical condition to the definition of approximately inner one-parameter groups in order to make the definition symmetric in a certain sense. (In known examples, the additional condition is automatically satisfied.) Consider the following possible additional properties:

* Supported by Odense University, Denmark 
(2) There is a dense subset $\mathscr{S}$ of $\mathfrak{A}$ such that the sequence of elements $\left(\left[h_{n}, a\right]\right)$ is Cauchy for all $a \in \mathscr{S}$, i.e., for all $a \in \mathscr{S}\left\|\left[h_{n}-h_{m}, a\right]\right\| \rightarrow 0$ as $n, m \rightarrow \infty$. (Note that $\mathscr{S}$ may be any dense subset. It need not be a core!)

(3) For all, $a \in \mathfrak{A}$ and $t \in \mathbb{R}$

$\left\|e^{i t\left(h_{n}-h_{m}\right)} a e^{-i t\left(h_{n}-h_{m}\right)}-a\right\| \rightarrow 0$ as $n, m \rightarrow \infty$.

(4) Let $\delta$ denote the infinitesimal generator for $\alpha^{t}$, and let $\delta_{n}$ be the inner derivation given by

$\delta_{n}(a)=\operatorname{ad} i h_{n}(a)=\left[i h_{n}, a\right]=i h_{n} a-i a h_{n}$ for $a \in \mathfrak{U}$.

Let $e^{t\left(\delta-\delta_{n}\right)}$ denote the strongly continuous one-parameter group of *-automorphisms generated by the perturbed derivation $\delta-\delta_{n}$ (see Lemma 1). Then for $a \in \mathfrak{A}$, and $t \in \mathbb{R}$ we have

$\left\|e^{t\left(\delta-\delta_{n}\right)}(a)-a\right\| \rightarrow 0 \quad$ as $n \rightarrow \infty$.

We show below that $(2) \Rightarrow(3)$. We verify in the proof of Theorem 1 that if (1) and (2) are satisfied for a one-parameter group $\alpha^{t}$ and an approximating sequence $\left(h_{n}\right)$, then (4) is satisfied as well for the same approximating sequence. However, (2) and therefore (3) may be satisfied even when no one-parameter group $\alpha^{t}$ exists. To see this we only have to note that (2) is trivially satisfied for all normal derivations.

Combining results of Sakai [13, Thm. 1], of Bratteli-Robinson [2, Thm. 11], and of Elliot [4] one can show in fact that (2) is satisfied for all closed *-derivations in UHF-algebras: For given a closed *-derivation $\delta$ in a UHF-algebra $\mathfrak{A}$, there is an increasing nest of finite type I subfactors $\left(\mathfrak{U}_{n}\right)$ contained in the domain of $\delta$ and with $\mathscr{S}=\bigcup_{n} \mathfrak{X}_{n}$ dense in $\mathfrak{A}$. There are elements $h_{n}=h_{n}^{*} \in \mathfrak{U}$ such that $\delta(a)=\left[i h_{n}, a\right]$ for $a \in \mathfrak{U}_{n}$. For every element $a \in \mathscr{S}$ the sequence $\left(\left[h_{n}, a\right]\right)$ is then trivially convergent.

Only the weakest of the three additional properties, number 4, is relevant for the proof of our main result. But properties (2) and (3) seem easier to check in applications where the $h_{n} s$ are known, but not $\delta$. Our proof applies to the cases where (1) is satisfied in combination with one of the three additional conditions for the same sequence $h_{n}$.

For quantum lattice systems with short range interaction the dynamics is given by approximately inner one-parameter groups of *-automorphisms [9, with references]. Condition (2), and therefore (3) and (4) as well, is clearly also satisfied for the quantum lattice systems [12, p. 193].

Proof that $(2) \Rightarrow(3)$. Let $\left(h_{n}\right)$ be a sequence and $\mathscr{S}$ a dense subset of $\mathfrak{U}$ satisfying condition (2). Let $\delta_{n}$ be the inner derivation ad $\left(i h_{n}\right)$ for each $n$. Since $\delta_{n}-\delta_{m}$ ad $i\left(h_{n}-h_{m}\right)$, the resolvents $R_{n m}^{ \pm}=\left(I \pm\left(\delta_{n}-\delta_{m}\right)\right)^{-1}$ exist and are everywhere defined contractions of $\mathfrak{A}$. For $a \in \mathscr{S}$ we have

$$
\begin{aligned}
& \left\|\left(I \pm\left(\delta_{n}-\delta_{m}\right)\right)^{-1}(a)-a\right\| \\
& \quad=\left\|\left(I \pm\left(\delta_{n}-\delta_{m}\right)\right)^{-1}\left\{a-\left(I \pm\left(\delta_{n}-\delta_{m}\right)\right) a\right\}\right\| \\
& \quad \leqq\left\|\left(\delta_{n}-\delta_{m}\right)(a)\right\| \rightarrow 0 \text { as } n, m \rightarrow \infty .
\end{aligned}
$$


It follows that $R_{n m}^{ \pm} \rightarrow I$ on the dense linear span of $\mathscr{S}$. But since $\left\|R_{n m}^{ \pm}\right\| \leqq 1$ that implies strong convergence of $\left(R_{n m}^{ \pm}\right)$to the identity everywhere. This implies in turn by the Trotter-Kato Theorem that condition (3) is satisfied. Note that

$$
e^{t\left(\delta_{n}-\delta_{m}\right)}(a)=e^{i t\left(h_{n}-h_{m}\right)} a e^{-i t\left(h_{n}-h_{m}\right)}
$$

and $e^{t\left(\delta_{n}-\delta_{m}\right)} \rightarrow I$. Moreover the convergence in (3) is uniform for $t$ in compact subintervals of the real line.

We conjecture that (3) is in fact a consequence of the defining property (1) for approximately inner one-parameter groups, and make the following definition to be used in the sequel.

Definition 1. A strongly continuous one-parameter group $\alpha^{t}$ of *-automorphism's of $\mathfrak{A}$ is said to be approximately inner if there is a sequence of hermitian elements $\left(h_{n}\right)$ in $\mathfrak{A}$ satisfying (1) and (3).

Since (2) $\Rightarrow(3)$, it is not hard to show that the one-parameter groups $\alpha^{t}$ generated by inner limit derivations are in fact approximately inner. (Such one-parameter groups were first constructed and examined in [9, Thm. 4.2]. We say with [2] that a closed derivation $\delta$ is an inner limit derivation if there is a sequence $\left(\delta_{n}\right)$ of inner derivations and a core $C$ for $\delta$ such that $\delta_{n}(a) \rightarrow \delta(a)$ as $n \rightarrow \infty$ for $a \in C$.) The proof of this fact is formally identical to the proof in [9, Thm. 4.2] for the special case where $\delta$ is the closure of a normal derivation in a UHF algebra.

\section{Statement of the Theorem and Some Applications}

Let $\alpha^{t}$ be a strongly continuous one-parameter group of *automorphisms of a $C^{*}$-algebra $\mathfrak{A}$. A state $\omega$ on $\mathfrak{H}$ is said to be a KMS state for inverse temperature $\beta=1 / k T$ with respect to $\alpha^{t}$ if for every pair of elements $a, b \in \mathfrak{A}$ there is a function $F(z)=F_{a, b, \beta}(z)$ which is holomorphic in the open strip $0<\operatorname{Im}(z)<\beta$, while continuous and bounded on the corresponding closed strip, which satisfies the KMS boundary conditions

$$
F(t)=\omega\left(a \alpha^{t}(b)\right) \quad \text { and } \quad F(t+i \beta)=\omega\left(\alpha^{t}(b) a\right)
$$

for all $t \in \mathbb{R}$.

It is known [16] that KMS states are automatically time-invariant, i.e., satisfy $\omega\left(\alpha^{t}(a)\right)=\omega(a)$.

Theorem 1. Let $\beta \neq 0$ be given, and suppose that there is at least one KMS state $\omega$ for inverse temperature $\beta$ with respect to an approximately inner one-parameter group $\alpha^{t}$ of *-automorphisms of a $C^{*}$-algebra $\mathfrak{A}$ with unit.

Then there is a sequence of states $\left(\omega_{n}\right)$ such that 1) each $\omega_{n}$ is a vector state w.r.t. $\omega$ in the GNS representation determined by $\omega$, and 2) all the accumulation points of $\left(\omega_{n}\right)$ are trace states $\tau$ on $\mathfrak{A}[$ i.e., $\tau(a b)=\tau(b a), \tau(I)=1]$. (Accumulation points exist since the state space is weak *-compact.) 3) If 2 t is separable then each trace state considered in 2) is a weak *-limit of a subsequence of $\left(\omega_{n}\right)$.

Recently Kruszynski [6] considered the special case of commutative derivations. He assumes that each $h_{n}$ in the approximating sequence is time invariant, i.e., $\alpha^{t}\left(h_{n}\right)=h_{n}$ for all $t$. He proves 1) and 2) for those derivations and asks whether 
the assumption on commutativity of the generator can be omitted. Our theorem solves this problem to the affirmative. We are grateful to Professor Sakai for bringing [6] to our attention and suggesting the desirability of an extension of Kruszynski's result.

Corollary 1. If KMS states exist for one value of inverse temperature, then they exist for all temperatures.

Proof. Under the assumption of the corollary it follows by Theorem 1 that $\mathfrak{A}$ has at least one trace state. But then the Powers-Sakai Theorem [9, Thm. 3.2] implies that KMS states exist for all temperatures.

Remark. Strictly speaking, only property (4), not (1), is needed for the proof of the conclusion of Theorem 1.

Remark. In physical models of statistical mechanics the dynamics is often given on UHF algebras, or closed *-subalgebras of such. In those cases the existence of a trace (even unique) is obvious. But from the point of view of an axiomatic theory and for other applications $[7,12]$ one is faced with $C^{*}$-algebras which are comple-

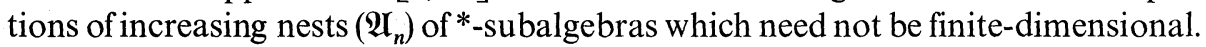
Such algebras generally do not have trace states.

Perhaps the theorem may also be viewed as a $C^{*}$ analog of the theorem of Takesaki which states that for a given faithful normal state on a von Neumann algebra, the corresponding modular automorphism group is inner iff the algebra is semifinite [14].

\section{Gentle Perturbations and Stability of the One-Parameter Groups}

Let $\alpha^{t}$ be an approximately inner one-parameter group of $*$-automorphisms of a $C^{*}$-algebra $\mathfrak{U}$, and let $\delta$ be the corresponding infinitesimal generator. Let $\left(h_{n}\right)$ be an approximating sequence of hermitian elements for $\alpha^{t}$ [i.e., satisfying (1) and (3)], and let $k=k^{*}$ be arbitrary in $\mathfrak{A}$. Let $\delta_{k}$ be the corresponding inner *-derivation $\delta_{k}=\operatorname{ad}(i k)$. Then $\delta+\delta_{k}$ is an unbounded *-derivation with the same domain as $\delta$. By semigroup theory we know that $\delta+\delta_{k}$ generates a strongly continuous oneparameter group of *-automorphisms. We denote this gently perturbed group by $\alpha_{k}^{t}$ and show that $\alpha_{k}^{t}$ is in fact approximately inner with $\left(h_{n}+k\right)$ as an approximating sequence. It turns out that all the properties (1) through (4) listed in the introduction are stable under the gentle perturbations $\delta_{k}$.

It is known [1] that $\alpha_{k}^{t}$ has KMS states for a given inverse temperature $\beta$ if and only if $\alpha^{t}$ has such states for the same $\beta$. If $k$ is also sufficiently analytic, then there is a useful formula (5) which for given $\beta$ connects the perturbed and the unperturbed KMS states.

We finally need the fact that small perturbations of the approximating sequence $\left(h_{n}\right)$ do not affect the limiting one-parameter group $\alpha^{t}$. That is, if $\left(k_{n}\right)$ is another sequence of hermitian elements such that $\left\|k_{n}-h_{n}\right\| \rightarrow 0$, then $\left(k_{n}\right)$ is also an approximating sequence for $\alpha^{t}$. Since the entire analytic elements for $\delta$ are always norm dense, this means that we may assume without loss of generality that the elements $h_{n}$ in an approximating sequence are entire analytic. 
Some of the results listed above, and proved below, are known but not exactly in the form and generality that we need.

Lemma 1. As above let $\alpha^{t}$ be an approximately inner one-parameter group with infinitesimal generator $\delta$ and approximating sequence $\left(h_{n}\right)$ of hermitian elements.

Let $k=k^{*} \in \mathfrak{U}$ be arbitrary. Then the one-parameter group $\alpha_{k}^{t}$ generated by $\delta+\delta_{k}$ is approximately inner, and $\left(h_{n}+k\right)$ is an approximating sequence of hermitian elements for this group.

Suppose $\alpha^{t}$ has a KMS state $\omega$ for a given inverse temperature. Then $\alpha_{k}^{t}$ has a $K M S$ state $\omega_{k}$ for the same inverse temperature. If $k$ is entire analytic, then there is an invertible element $v_{k} \in \mathfrak{U}$ such that

(5) $\omega_{k}(a)=\omega\left(v_{k}^{*} a v_{k}\right) / \omega\left(v_{k}^{*} v_{k}\right.$ for all $a \in \mathfrak{A}$.

Proof. Part of the lemma is known for one-parameter groups of *-automorphisms of special $C^{*}$-algebras which have a unique trace state [10]. But if a trace is not a priori assumed or known to exist, then the proof must proceed along different lines. The last conclusion of the lemma holds for one-parameter groups that are not necessarily approximately inner.

It follows from semigroup theory and from [1] that the infinite series

$$
\sum_{p=0}^{\infty} \int_{0 \leqq s_{1} \leqq \ldots \leqq s_{p} \leqq t} \alpha^{s_{1}} \delta_{k} \alpha^{s_{2}-s_{1}} \delta_{k} \ldots \delta_{k} \alpha^{t-s_{p}} d s_{1} \ldots d s_{p}
$$

is absolutely convergent for all $t \geqq 0$ and defines a strongly continuous one-

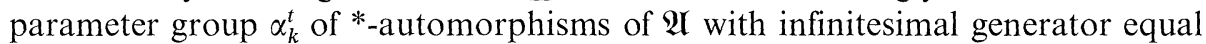
to $\delta+\delta_{k}$. Using the definition of $\delta_{k}$ and the automorphisms property of $\alpha^{t}$ one shows that there is a one-parameter "multiplier" of *-automorphisms $\eta(t, k, \alpha)$ such that

(6) $\alpha_{k}^{t}=\eta(t, k, \alpha) \alpha^{t}$.

In fact

(7) $\eta(t, k, \alpha)=\sum_{p=0}^{\infty} i^{p} \int_{0 \leqq s_{1} \leqq \ldots \leqq s_{p} \leqq t} \operatorname{ad}\left(\alpha^{s_{1}}(k)\right) \ldots \operatorname{ad}\left(\alpha^{s_{p}}(k)\right) d s_{1} \ldots d s_{p}$.

Similarly, the one-parameter group $\sigma_{n}^{t}$ generated by $\operatorname{ad}\left(i h_{n}\right)+\operatorname{ad}(i k)=\operatorname{ad} i\left(h_{n}+k\right)$ is given by $\sigma_{n}^{t}=\eta\left(t, k, \alpha_{n}\right) \alpha_{n}^{t}$. Therefore we have

(8) $\left\|\alpha_{k}^{t}(a)-\sigma_{n}^{t}(a)\right\| \leqq\left\|\eta(t, k, \alpha)\left\{\alpha^{t}(a)-\alpha_{n}^{t}(a)\right\}\right\|$

$$
\begin{aligned}
& +\left\|\left\{\eta(t, k, \alpha)-\eta\left(t, k, \alpha_{n}\right)\right\} \alpha_{n}^{t}(a)\right\| \\
& \leqq\left\|\alpha^{t}(a)-\alpha_{n}^{t}(a)\right\|+\left(2 t A_{n, t}\right) \exp (2 t\|k\|)\|a\|,
\end{aligned}
$$

where $A_{n, t}=\sup \left\{\left\|\alpha^{s}(k)-\alpha_{n}^{s}(k)\right\|: 0 \leqq s \leqq t\right\}$.

If $\alpha_{n}^{s \rightarrow \alpha^{s}}$ in the strong operator topology, uniformly for $s$ in compacta, then the right hand side of (8) tends to zero as $n \rightarrow \infty$, again uniformly for $t$ in compact subintervals of the line. It is self evident that each of the conditions (2) through (4) is satisfied for the sequence $\left(h_{n}\right)$ if and only if it is satisfied for $\left(h_{n}+k\right)$.

Suppose now that $\omega$ is a KMS state for inverse temperature $\beta$ with respect to $\alpha^{t}$, and that $k=k^{*}$ is entire analytic. The function $t \rightarrow \alpha^{t}(k)$ then has an analytic 
continuation to the whole complex plane. It is given for all $z$ by an absolutely convergent power series $\alpha^{z}(k)=\sum z^{p} / p ! \delta^{p}(k)$. If we put

(9) $v_{k}=\sum_{p=0}^{\infty}(-1)^{p} \int_{0 \leqq s_{1} \leqq \ldots \leqq s_{p} \leqq \beta / 2} \alpha^{i s_{1}}(k) \ldots \alpha^{i s_{p}}(k) d s_{1} \ldots d s_{p}$,

then $v_{k}$ is invertible, and the state $\omega_{k}(a)=\omega\left(v_{k}^{*} a v_{k}\right) / \omega\left(v_{k}^{*} v_{k}\right)$ satisfies the KMS boundary for the same inverse temperature with respect to the perturbed oneparameter group $\alpha_{k}^{t}$. The formula (9) is due to Araki [1] and Robinson [11], as is the non-trivial verification of the KMS condition for $\omega_{k}$. (If $H$ denotes the formal Hamiltonian for $\alpha^{t}$, then formally $v_{k}=e^{-\beta(H+k) / 2} e^{\beta H / 2}$.) It is of interest that the element $v_{k}$ can be shown to be invertible.

The existence of a KMS state $\omega_{k}$ for arbitrary $k=k^{*}$ which is not entire analytic is proven by Araki [1]. Such general $\omega_{k}$ are typically weak *-accumulation points of the set of states given by (5). In our theorem we approximate with vector states of the form (5) and thus obtain more explicit information.

Lemma 2. Let $\alpha^{t}$ be an approximately inner dynamical one-parameter group, and let $\left(h_{n}\right)$ be an approximating sequence of hermitian elements. If $\left(k_{n}\right)$ is another sequence of hermitian elements such that $\left\|h_{n}-k_{n}\right\| \rightarrow 0$, then $\left(k_{n}\right)$ is also an approximating sequence for $\alpha^{t}$.

Proof. For an element $l=l^{*} \in \mathfrak{U}$ we denote the ${ }^{*}$-derivation $\operatorname{ad}(i l)$ by $\delta_{l}$ and the corresponding *-automorphism group

$$
e^{t \delta_{l}}(a)=e^{i t l} a e^{-i t l}
$$

by $\alpha_{l}^{t}$. Put $c_{n}=k_{n}-h_{n}$. By the perturbation formulas (6) and (7) we have

$$
\alpha_{k_{n}}^{t}=\eta\left(t, c_{n}, \alpha_{h_{n}}\right) \alpha_{h_{n}}^{t},
$$

and

$$
\left\|\eta\left(t, c_{n}, \alpha_{h_{n}}\right) x-x\right\| \leqq\left(\exp \left(2\left\|c_{n}\right\| t\right)-1\right)\|x\|
$$

for all $n=1,2, \ldots, t \geqq 0$ and $x \in \mathfrak{U}$. Consequently

$$
\left\|\alpha_{k_{n}}^{t}(a)-\alpha_{h_{n}}^{t}(a)\right\| \leqq\left(\exp \left(2\left\|c_{n}\right\| t\right)-1\right)\|a\| .
$$

The right hand side tends to zero for every $a \in \mathfrak{U}$ as $n \rightarrow \infty$, the convergence being uniform for $t$ in compact intervals. (The case $t \leqq 0$ goes the same way.) It follows that $\alpha_{k_{n}}^{t}(a) \rightarrow \alpha^{t}(a)$.

The symmetry property (3) for the sequence $k_{n}=h_{n}+c_{n}$ can be verified the same way. Since by assumption

$$
\alpha_{h_{n}-h_{m}}^{t}(a) \rightarrow a \text { for } n, m \rightarrow \infty \text {; }
$$

it is enough to check that $\left\|\alpha_{k_{n}-k_{m}}^{t}(a)-\alpha_{h_{n}-h_{m}}^{t}(a)\right\| \rightarrow 0$ as $n, m \rightarrow \infty$. This follows in turn from the estimate

$$
\left\|\alpha_{k_{n}-k_{m}}^{t}(a)-\alpha_{h_{n}-h_{m}}^{t}(a)\right\| \leqq\left(\exp \left(2\left\|c_{n}-c_{m}\right\| t\right)-1\right)\|a\| .
$$

This time we use the perturbation multiplier formula for the perturbations $\delta_{k_{n}-k_{m}}=\delta_{h_{n}-h_{m}}+\delta_{c_{n}-c_{m}}$. 


\section{Proof of Theorem 1}

Let $\left(\alpha^{t},\left(h_{n}\right), \mathfrak{A}, \omega, \beta\right)$ be as in the assumptions of the theorem. Since the entire analytic elements are norm dense (see [2] II, or [8]) we may assume without loss of generality, using Lemma 2, that the elements in the approximating sequence $\left(h_{n}\right)$ are entire analytic. Let $\left(v_{n}\right)$ be the corresponding sequence of elements such that $v_{n}$ is defined from $-h_{n}$ by the formula (9) for each $n$. Then $\omega_{n}(a)=\omega\left(v_{n}^{*} a v_{n}\right) /$ $\omega\left(v_{n}^{*} v_{n}\right), a \in \mathfrak{A}$, defines a KMS state for inverse temperature $\beta$ with respect to the one-parameter group $\sigma_{n}^{t}=e^{t\left(\delta-\delta_{n}\right)}$ generated by $\delta-\delta_{n}$ with $\delta_{n}=\operatorname{ad}\left(i h_{n}\right)$ for each $n$.

We shall need that $\sigma_{n}^{t} \rightarrow I$ in the strong operator topology and uniformly for $t$ in compact subintervals. [This is the only point where the symmetry condition (3) is needed.] The uniform convergence on compacta is an automatic consequence of pointwise convergence by the Trotter-Kato Theorem. Using Lemma 1, we first note that for each positive integer $m$ (fixed) the sequence of inner automorphisms $\left\{e^{t\left(\delta_{n}-\delta_{m}\right)}\right\}$ converges in the strong operator topology to $e^{t\left(\delta-\delta_{m}\right)}$ as $n \rightarrow \infty$. The desired conclusion (4): $\sigma_{m}^{t}=e^{t(\delta-\delta m)} \rightarrow I$ follows from this combined with (3) and a simple $2 \varepsilon$ argument.

The sequence of states $\left(\omega_{n}\right)$ must have at least one weak *-accumulation point because the state space of $\mathfrak{A}$ is weak *-compact. Let $\tau$ be such an accumulation point. We use the arguments from [9] combined with the considerations above to show that $\tau$ must be a trace state.

In the rest of the proof $a$ and $b$ are arbitrary but fixed elements in $\mathfrak{A}$. There are corresponding analytic functions $F_{n}(z)$ in $0<\operatorname{Im}(z)<\beta$ which extend to continuous and bounded functions on the closed strip $0 \leqq \operatorname{Im}(z) \leqq \beta$, and satisfy the KMS boundary conditions with respect to $\omega_{n}$ for each $n$. That is

$$
F_{n}(t)=\omega_{n}\left(a \sigma_{n}^{t}(b)\right) \text { and } F_{n}(t+i \beta)=\omega_{n}\left(\sigma_{n}^{t}(b) a\right)
$$

for all $t \in \mathbb{R}$. The boundary functions $f_{n}(t)=F_{n}(t)$ and $g_{n}(t)=F_{n}(t+i \beta)$ are both continuous, and in fact uniformly bounded: $\left|f_{n}(t)\right| \leqq\|a\|\|b\|,\left|g_{n}(t)\right| \leqq\|a\|\|b\|$ for all $n=1,2, \ldots$ and $t \in \mathbb{R}$.

Since $F_{n}(z)$ is continuous up to the boundary, the Dirichlet problem of the strip $0 \leqq \operatorname{Im}(z) \leqq \beta$ with boundary conditions $f_{n}(t)$ and $g_{n}(t)$ for each $n$ has the unique solution $F_{n}(z)$. It is given by an integral representation in terms of the Greens function of the domain $0 \leqq \operatorname{Im}(z) \leqq \beta$ [5, Sec. 18.2]

$$
F_{n}(z)=\int_{-\infty}^{\infty}\left(C(t, z) f_{n}(t)+D(t, z) g_{n}(t)\right) d t
$$

where $C(t, z) \geqq 0, D(t, z) \geqq 0$, and $\int_{-\infty}^{\infty}(C(t, z)+D(t, z)) d t \equiv 1$. We have supplied a few considerations leading up to this by now standard integral formula for harmonic functions in a strip, also used in [6] and [9].

Now take a subsequence $\left(\omega_{n_{j}}\right)$ of $\left(\omega_{n}\right)$ such that $\omega_{n_{j}}(a b) \rightarrow \tau(a b)$ and $\omega_{n_{j}}(b a) \rightarrow \tau(b a)$. In the case where $\mathfrak{A}$ is separable we may take the subsequence $\left(\omega_{n_{j}}\right)$ such that $\omega_{n_{j}} \rightarrow \tau$ pointwise, but in general the subsequence will depend on the points $a$ and $b$. We must show that the corresponding subsequence $\left(F_{n_{j}}(z)\right)$ of analytic functions has a limit $F(z)$ which is analytic in $0<\operatorname{Im}(z)<\beta$, and continuous and bounded on the closure. 
Note first that $f_{n_{j}}(t) \rightarrow \tau(a b)$ and $g_{n_{j}}(t) \rightarrow \tau(b a)$ as $j \rightarrow \infty$, the convergence being uniform for $t$ in compact subintervals. For $\left(f_{n_{j}}\right)$ this follows from

$$
\left|\omega_{n_{j}}\left(a \sigma_{n_{j}}^{t}(b)\right)-\tau(a b)\right| \leqq\|a\|\left\|\sigma_{n_{j}}^{t}(b)-b\right\|+\left|\omega_{n_{j}}(a b)-\tau(a b)\right| .
$$

The first term on the right hand side tends to zero since $\sigma_{n}^{t}(b) \rightarrow b$ as observed above, and the second tends to zero by assumption. From the Dominated Convergence Theorem applied to (10), or the fact that the Dirichlet problem is well posed, we now conclude that the sequence $\left(F_{n_{j}}(z)\right)$ has a limit $F(z)$ of the form specified above. The boundary values are $F(t)=\tau(a b)$ and $F(t+i \beta)=\tau(b a)$. Such boundary values are possible only when $F$ is constant. Therefore $\tau(a b)=\tau(b a)$.

Acknowledgements. It is a pleasure to thank Professor S. Sakai for showing us the preprint [6] (which proves a version of our Theorem 1 for the special case of commutative derivations) and for suggesting the desirability of an extension of Kruszynski's result. We are also grateful to Professor Sakai for constant encouragement and for reading the manuscript and making useful suggestions; to Professor C. Radin for helpful conversations; and to Professor R. V. Kadison for warm hospitality at the University of Pennsylvania.

\section{References}

1. Araki, H.: Pub. Res. Inst. Math. Sci. Kyoto 9, 165-209 (1973)

2. Bratteli, O., Robinson, D. W.: Commun. math. Phys. 42, 253-268 (1975); 46, 11-30 (1976)

3. Chernoff, P.: Product formulas, nonlinear semigroups, and addition of unbounded operators, Amer. Math. Soc. Memoirs 140, (1974)

4. Elliott, G.: Inventiones Math. 9, 253-269 (1970)

5. Hille, E.: Analytic function theory, Vol. II, New York: Ginn and Co. 1962

6. Kruszynski, P.: On the existence of KMS states for invariantly approximately inner dynamics, to appear in Bull. Acad. Pol. Sci.

7. Narnhofer, H., Robinson, D. W.: Commun. math. Phys. 41, 89-97 (1975)

8. Nelson, E.: Ann. Math. 70, 572-615 (1959)

9. Powers, R.T., Sakai, S.: Commun. math. Phys. 39, 273-288 (1975)

10. Radin, C.: Commun. math. Phys. 23, 189-198 (1971)

11. Robinson, D. W.: Proc. Varenna Summer School (1973)

12. Ruelle, D.: Statistical mechanics, New York: W. A. Benjamin Inc. 1969

13. Sakai,S.: On one-parameter subgroups of *-automorphisms on operator algebras and the corresponding unbounded derivations. Amer. J. Math. 98 (1976)

14. Takesaki, M.: Tomita's theory of modular Hilbert algebras and its applications, Lecture Notes in Mathematics, Vol. 128. Berlin-Heidelberg-New York: Springer 1970

15. Trotter, H.F.: Pacific J. Math. 8, 887-919 (1958)

16. Winnink, M.: Thesis, University of Gröningen, 1968

17. Yosida, K.: Functional analysis, 4th ed., Berlin-Heidelberg-New York: Springer 1974

Communicated by H. Araki

Received May 17, 1976; in revised form October 12, 1976 